\title{
Modelling the structure of the hot components in symbiotic binaries during outbursts
}

\author{
Zuzana Cariková and Augustin Skopal \\ Astronomical Institute, Slovak Academy of Sciences, \\ 05960 Tatranská Lomnica, Slovakia \\ email: zcarikova@ta3.sk, skopal@ta3.sk
}

\begin{abstract}
During outbursts in symbiotic binaries a neutral medium in the form of a flared disk develops around their hot stars. The appearance of the disks is most probably connected with the enhanced wind from the hot star. The wind is compressed towards the equatorial plane of the hot star due to its rotation. To test this idea we calculated the ionization boundaries in the enhanced wind and thus confirmed the possibility of the formation of the neutral disk-like zones around the hot stars in symbiotic binaries during outbursts.
\end{abstract}

Keywords. Stars: binaries: symbiotic, stars: winds: outflows

\section{Introduction}

Symbiotic stars are long-period interacting binary systems, which comprise a cool giant and a hot compact star, mostly a white dwarf. Modelling the spectral energy distribution (SED) of many symbiotic binaries showed that the temperature of the hot component during active phases (outbursts) is $\sim 10^{5} \mathrm{~K}$ in binaries with a low orbital inclination, while for systems with a high orbital inclination it decreases to $\sim 2 \times 10^{4} \mathrm{~K}$ (Skopal 2005). In both cases strong increase in the nebular emission was indicated. Therefore Skopal (2005) proposed that a neutral disk-like structure is created around the central hot star near the orbital plane during active phases. As a result in symbiotic binaries with high orbital inclination we observe the flared rim of the disk (i.e. warm pseudophotosphere), which radiates at significantly lower temperature than the central hot star. On the other hand, in symbiotic binaries with low orbital inclination we can observe the central hot star directly. Modelling the broad $\mathrm{H}_{\alpha}$ wings showed that during active phases the stellar wind from the hot star is enhanced, from $10^{-8} M_{\odot} \mathrm{yr}^{-1}$ during quiescent phases to $10^{-7}$ - $10^{-6} M_{\odot} \mathrm{yr}^{-1}$ during active phases (Skopal 2006).

We tested the idea of the formation of the neutral disk-like zones around the hot stars in symbiotic binaries during outbursts by calculating the ionization boundaries in the enhanced wind from the rotating hot star.

\section{Ionization structure in the compressed hot star wind}

Rotation of the hot star leads to a compression of the outflowing material towards the equatorial plane. The wind compression model was described by Bjorkman \& Cassinelli (1993) and Ignace et al. (1996). We calculated the ionization boundary in the compressed wind from the hot star by the equation of photoionization equilibrium. Figure 1 shows an example of the calculated ionization structure in the enhanced wind from the hot star during active phases. The H I/H II boundary was calculated according to Cariková \& Skopal (2012). 


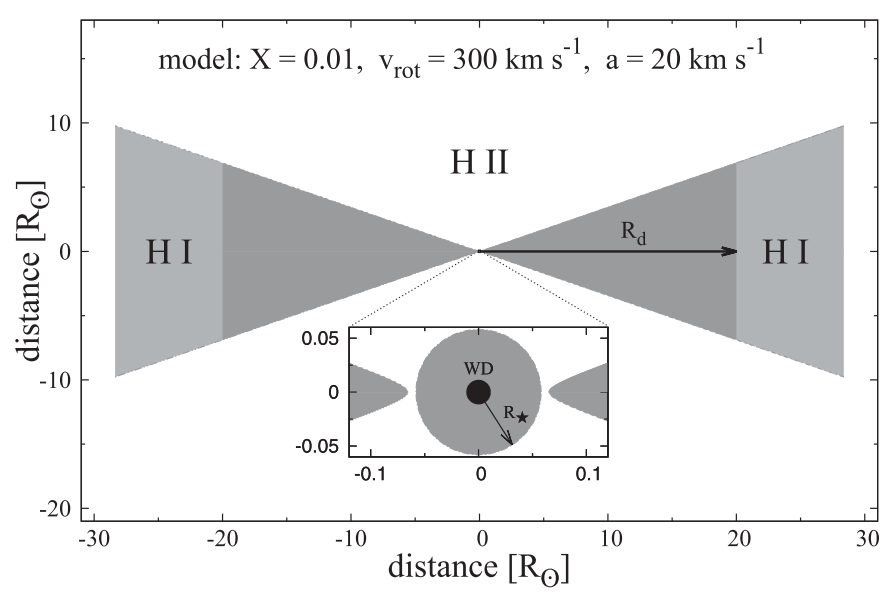

Figure 1. Ionization structure of the hot component during activity as seen on a cut perpendicular to the orbital plane containing the white dwarf. Warm pseudophotosphere which radiates at significantly lower temperature than the central hot star is located at the distance $R_{\mathrm{d}}$ (optically thick/thin boundary). Above and below this neutral disk-like zone there are the ionized regions of the hot star wind. Small figure shows the central hot star with radius $R_{\star}$, which is the source of the ionizing photons and WD (black circle), which is the source of the stellar wind. (adopted according to Cariková \& Skopal (2012))

\section{Conclusion}

We showed that during active phases the compression of the enhanced stellar wind from the rotating hot star towards the equatorial regions can lead to the creation of the neutral disk-like zone around the hot star (see Fig. 1). In symbiotic binaries with high orbital inclination we are looking through the neutral disk-like zone, whose optically thick boundary at the distance $R_{\mathrm{d}}$ from the central star simulates the warm pseudophotosphere radiating at significantly lower temperature than the central hot star.

We also calculated column densities of the neutral hydrogen atoms through the disklike zone above the warm pseudophotosphere, and emission measure of the ionized part of the hot star wind. We found that these values are in a good agreement with the observed quantities, and thus support our idea of creation of the neutral disk-like zones around the hot stars in symbiotic binaries during outbursts.

\section{Acknowledgement}

This research was supported by a grant of the Slovak Academy of Sciences, VEGA No. 2/0038/10. Z. C. acknowledges support from the IAU.

\section{References}

Bjorkman, J. E. \& Cassinelli, J. P. 1993, ApJ, 409, 429

Cariková, Z. \& Skopal, A. 2012, A\&A (in press)

Ignace, R., Cassinelli, J. P., \& Bjorkman, J. E. 1996, ApJ, 459, 671

Skopal, A. 2005, A\&A, 440, 995

Skopal, A. 2006, A\&A, 457, 1003 